\title{
Transfer Subspace Learning Model for Face Recognition at a Distance
}

\author{
Alwin Anuse \\ MIT, Pune ,India \\ Email: alwin.anuse@mitpune.edu.in \\ Nilima Deshmukh \\ AISSM'S IOT,India \\ Email: deshmukhnilima@gmail.com \\ Vibha Vyas \\ College of Engineering Pune,India \\ Email: vsv.extc@coep.ac.in
}

\begin{abstract}
Many machine learning algorithms work under the assumption that the training and testing data are drawn from the same distribution. However, in practice the assumption might not hold. Transfer subspace learning algorithms aims at utilizing knowledge gained in source domain to learn a task in target domain. The main objective of this work is to apply transfer subspace learning framework on face recognition task at a distance. In this paper we identify face recognition at distance as a transfer learning problem. We show that if the face recognition task is modeled as transfer learning problem, the overall classification rate is increased significantly compared to traditional brute force approach. We also discuss a data set which is unique and meant to advance this research. The novelty of this work lies in modeling face recognition task at distance as a transfer subspace learning problem.
\end{abstract}

Index Terms-Face recognition, Transfer subspace learning, KNN, independent and identically distributed.

\section{INTRODUCTION}

Many Machine learning algorithms assume that the training and testing data belong to same feature space and the same distribution [1]. However, in practice this is not the situation. The data may belong to different distribution, for e.g. in face recognition application, the face images may be taken under different illumination conditions, pose changes, expression changes etc. It is very difficult to maintain the same environmental conditions at the time of testing which were present during image acquisition for training task. The training data might not be available at the same time. The system has to be retrained if the data distribution changes. In many situations, it is expensive or impossible to collect the training data and retrained the system [1]. In such situations transfer learning approach is useful.
Transfer learning approach stores knowledge at the time of training and uses it at the time of testing. Transfer learning uses both labeled and unlabeled samples, similar to semi supervised learning. In semi supervised learning, the training and testing samples are usually independent and identically distributed (i.i.d) [3] and thus, the distribution of the training samples is consistent with that of testing samples. When labeled samples are available, auxiliary information is utilized in transfer learning. The auxiliary information may be in the form of sharing features from auxiliary tasks [4], data from auxiliary domains [5]. By assuming that both source and target modality are accessible in the training phase, knowledge is transferred in the multimodal transfer learning. For e.g. in Face recognition one might have near infrared images as source and visible images as target modality [11]. Liu Yang and Etal showed that text-to-image transfer learning can be done in noisy environment [12]. Transfer learning is been used in regression, classification and unsupervised learning [13].

Lot of research is going on developing Face recognition algorithms which are invariant to pose [14], expressions [15], illumination [16] and distance [17]. In this paper we have addressed the problem of face recognition at a distance. The contribution of this research work are:

a. Use of transfer learning model for face recognition at a distance

b. Novel dataset which is developed and meant to advent this research.

\section{RELATED WORK}

Transfer subspace learning has advanced considerably since the work of Si Si et.al (2010), which we use our baseline. In their research [2], they have proposed Bregman divergence based regularization for transfer subspace learning which boost performance when training and testing samples are not independent and 
identically distributed. They performed their experiments on public datasets, e.g. YALE [6], FERET [7] etc. None of the dataset is meant for distance invariance experimentation .The dataset described by us meant exclusive for the experimentation on distance invariance. Many researchers have applied subspace learning to small scale applications like text classification, sensor network based localization, image classification [4-5].Various application of transfer subspace learning are explained in [10].

\section{TRANSFER SUBSPACE LEARNING FRAMEWORK}

Let there be $\mathrm{m}$ training and $\mathrm{n}$ testing samples, which belongs to a high dimensional space $\mathrm{R}^{\mathrm{S}}$. Any subspace learning algorithm can find a low dimensional space $\mathrm{R}^{\mathrm{s}}$, wherein we get separation among samples from different classes. If $x$ is the feature vector such that $x \in R^{S}$, then there exists linear function $y=V^{T} x$, wherein $V \epsilon R^{\text {Sxs }}$ and $y \in R^{\mathrm{s}}$. The linear function can be obtained from

$$
V=\arg \min F(V)
$$

Subject to $\mathrm{V}^{\mathrm{T}} \mathrm{V}=\mathrm{I}$. The objective function $\mathrm{F}(\mathrm{V})$ is designed to minimize the classification error. The traditional subspace learning framework (1) will perform well only if training and testing samples are independent and identically distributed. However, sometimes the distribution of the training samples $\mathrm{P}_{\mathrm{m}}$ and that of testing samples $\mathrm{P}_{\mathrm{n}}$ is different. Under such conditions, the subspace learning framework (1) will fail. To address this problem one can use Bregman divergence-based regularization $\mathrm{D}_{\mathrm{V}}\left(\mathrm{P}_{\mathrm{m}} \| \mathrm{P}_{\mathrm{n}}\right)$, which measures the distance between the distributions of the training and testing samples in a projected subspace V. Accordingly, the framework in (1) is modified as given in eqn (2)

$$
V=\arg F(V)+\alpha D_{V}\left(P_{m} \| P_{n}\right)
$$

Subject to $\mathrm{V}^{\mathrm{T}} \mathrm{V}=\mathrm{I}$. Regularization parameter $\alpha$ controls the trade-off between $F(V)$ and $D_{V}\left(P_{m} \| P_{n}\right)$. Gradient descent algorithm can be used to obtain the solution of (2), i.e.

$$
\begin{gathered}
V(\text { new })=V(\text { old })- \\
\mu\left(\frac{\partial F(V)}{\partial V}+\alpha \frac{\partial D_{V}\left(P_{m} \| P_{n}\right)}{\partial V}\right)
\end{gathered}
$$

Where $\mu$ is the learning rate.

\section{A. Framework of Transfer Subspace learning(TSL)} applied to Principal Component Analysis (PCA)

There are many popular subspace learning algorithms like unsupervised principle component analysis (PCA), supervised linear discriminant analysis (LDA) and locality preserving projection (LPP). Projection of data by linear transformation technique is a key concept in all these algorithms.

PCA projects the high dimensional data to lower dimensional space by capturing maximum variance [8].
PCA projection matrix maximizes the trace of the total scatter matrix

$$
V=\arg \max t r\left(V^{T} A V\right)
$$

Subject to $\mathrm{V}^{\mathrm{T}} \mathrm{V}=\mathrm{I}$. A is the autocorrelation matrix of training samples. $\mathrm{F}(\mathrm{V})$ of PCA is given by (5)

$$
\begin{gathered}
F(V)=-\operatorname{tr}\left(V^{T} A V\right) \\
\frac{\partial F(V)}{\partial V}=-2 A V
\end{gathered}
$$

\section{ALGORITHM}

In subspace learning algorithm, high dimensional data is projected into a low dimensional subspace preserving specific statistical properties. Fisher linear discriminative analysis (FLDA), minimizes the trace ratio between the within class and between the class scatter [18]. Locality preserving projection (LPP) preserves the local geometry of samples [19]. Principal component analysis (PCA) is an unsupervised method that projects the high dimensional data to lower dimensional space by capturing maximum variance. PCA steps are explained in section IV (A). If the training and testing samples are not independent and identically distributed, PCA gives very poor performance. Transfer principal component analysis (TPCA) learning algorithm takes into account the distribution difference between the training and testing samples. TPCA steps are explained in section IV (B).

\section{A. PCA steps}

Step 1 Subtract the mean

From all the samples of training set, subtract the mean from each of the data dimensions.

Step 2 Calculate the covariance matrix

Step3 Calculate the eigen vectors and eigen values of the covariance matrix

Step 4 Choose components and form a feature vector

The eigen vector with the highest eigen value is the principle component of the data set. Feature vector is constructed by taking the eigen vectors that we want to keep from the list of eigen vectors.

Step5 Deriving new data set.

New data vector $\mathrm{y}=\mathrm{V} \mathrm{x}$, where $\mathrm{x}$ is old vector and $\mathrm{V}$ is the transformation matrix of PCA projection matrix made of eigen vectors.

\section{B. TPCA ( Transfer Principle Component Analysis) steps}

Step 1 Add new samples to the old data set.

Step 2 Choose the initial guess V

$\mathrm{V}$ learned from $\mathrm{F}(\mathrm{v})$ is a good initial guess.

Step 3 Choose the learning rate $\mu$ and regularization parameter $\alpha$.

These values should be greater than zero but less than or equal to one.

Step 4 Find the autocorrelation matrix of the samples in the dataset. 
Step 5 Update

Equation (3) subject to $\mathrm{V}^{\mathrm{T}} \mathrm{V}=\mathrm{I}$.

\section{DATASET}

For the experimentation, we constructed our own database. By varying the distance between camera and subject, the database was prepared. The distance was varied in steps of $15 \mathrm{~cm}$. We referred to distance of $15 \mathrm{~cm}$ a scale $\mathrm{S} 1,30 \mathrm{~cm}$ as $\mathrm{S} 2$ and $120 \mathrm{~cm}$ as $\mathrm{S} 8$ etc. The database contains 10000 images that includes 50 subjects .For every subject 25 images at a distance of 15 $\mathrm{cm}$ were taken. The database is under construction. Sample images are shown in Fig1.

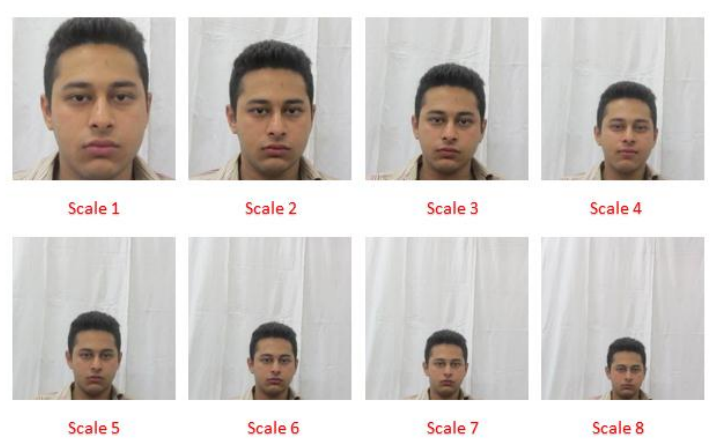

\section{EXPERIMENTATION}

KNN ( $\mathrm{K}$ nearest Neighbors) [9] classifier is been trained with PCA features for different subspaces and the classification rates on same scale and cross scale is found. $\mathrm{KNN}$ classifier is also been trained and tested with Transfer PCA features for different subspace dimensions. The results of the same are shown in table 1-6. Brute force approach is also used to train KNN. In Brute force approach the KNN is trained with samples taken at two distances. Results of brute force method are shown in table 7.

Regularization parameter was heuristically set to 0.5 . The learning rate parameter was initially set to 1 and then decreased to 0.3 . The nearest neighbor rule is used for classification. It is essential to have one reference image for each testing class. In the training stage no labeling information is available. The labeling information of reference images is available only for classification in the testing stage. Distance between every reference image and testing image is calculated for predicting the label of the testing image as that of the nearest reference image.

Fig.1. Sample Images in Database

\section{RESULtS}

Table 1. PCA with $10 \times 10$ subspace

\begin{tabular}{|c|c|c|c|c|c|c|c|c|}
\hline \multicolumn{9}{|c|}{ KNN classifier trained with PCA Features with $10 \times 10$ subspace dimensions } \\
\hline \multirow[t]{2}{*}{ Training } & \multicolumn{8}{|c|}{ Testing } \\
\hline & S1 & $S 2$ & $S 3$ & $S 4$ & S5 & S6 & S7 & S8 \\
\hline S1 & 75 & 10 & 9 & 15 & 4 & 8 & 9 & 7 \\
\hline S2 & 17 & 78 & 18 & 12 & 11 & 8 & 10 & 12 \\
\hline S3 & 10 & 10 & 74 & 18 & 11 & 17 & 17 & 12 \\
\hline$S 4$ & 12 & 13 & 9 & 76 & 8 & 9 & 12 & 13 \\
\hline S5 & 5 & 6 & 7 & 22 & 78 & 24 & 17 & 12 \\
\hline S6 & 15 & 13 & 15 & 10 & 19 & 79 & 22 & 15 \\
\hline S7 & 13 & 15 & 12 & 11 & 10 & 13 & 78 & 13 \\
\hline$S 8$ & 10 & 6 & 12 & 11 & 15 & 25 & 26 & 88 \\
\hline
\end{tabular}

Table 2. TPCA with $10 \times 10$ subspace

\begin{tabular}{|c|c|c|c|c|c|c|c|c|}
\hline \multirow[t]{2}{*}{ Training } & \multicolumn{8}{|c|}{ Testing } \\
\hline & S1 & $S 2$ & $S 3$ & S4 & $S 5$ & S6 & $S 7$ & S8 \\
\hline$S 1$ & 78 & 60 & 75 & 70 & 80 & 78 & 85 & 84 \\
\hline$S 2$ & 88 & 82 & 84 & 85 & 75 & 80 & 82 & 85 \\
\hline S3 & 45 & 90 & 91 & 90 & 90 & 85 & 88 & 85 \\
\hline$S 4$ & 40 & 95 & 95 & 90 & 78 & 92 & 92 & 92 \\
\hline$S 5$ & 46 & 55 & 72 & 72 & 82 & 85 & 84 & 85 \\
\hline S6 & 50 & 95 & 95 & 95 & 97 & 88 & 97 & 96 \\
\hline S7 & 48 & 90 & 83 & 84 & 86 & 82 & 82 & 80 \\
\hline$S 8$ & 45 & 92 & 93 & 92 & 93 & 94 & 94 & 95 \\
\hline
\end{tabular}


Table 3. PCA with $20 \times 20$ subspace

\begin{tabular}{|c|c|c|c|c|c|c|c|c|}
\hline \multicolumn{9}{|c|}{ KNN classifier trained with PCA Features with $20 \times 20$ subspace dimensions } \\
\hline \multirow[t]{2}{*}{ Training } & \multicolumn{8}{|c|}{ Testing } \\
\hline & S1 & $S 2$ & S3 & $S 4$ & S5 & S6 & S7 & S8 \\
\hline S1 & 82 & 14 & 4 & 5 & 8 & 9 & 12 & 12 \\
\hline$S 2$ & 14 & 80 & 18 & 12 & 16 & 10 & 12 & 13 \\
\hline S3 & 14 & 16 & 80 & 20 & 18 & 18 & 10 & 15 \\
\hline$S 4$ & 7 & 10 & 13 & 85 & 16 & 10 & 12 & 10 \\
\hline$S 5$ & 11 & 10 & 8 & 9 & 87 & 10 & 11 & 16 \\
\hline S6 & 12 & 10 & 13 & 12 & 10 & 78 & 12 & 10 \\
\hline$S 7$ & 18 & 12 & 14 & 18 & 17 & 16 & 81 & 12 \\
\hline$S 8$ & 13 & 18 & 15 & 16 & 8 & 9 & 10 & 75 \\
\hline
\end{tabular}

Table 4. TPCA with $20 \times 20$ subspace

\begin{tabular}{|c|c|c|c|c|c|c|c|c|}
\hline \multicolumn{9}{|c|}{ KNN classifier trained with TPCA Features with $20 \times 20$ subspace dimensions } \\
\hline \multirow[t]{2}{*}{ Training } & \multicolumn{8}{|c|}{ Testing } \\
\hline & $S 1$ & $S 2$ & S3 & S4 & $S 5$ & S6 & $S 7$ & S8 \\
\hline$S 1$ & 85 & 92 & 90 & 75 & 84 & 98 & 98 & 97 \\
\hline$S 2$ & 97 & 82 & 75 & 97 & 48 & 94 & 97 & 98 \\
\hline$S 3$ & 50 & 55 & 83 & 45 & 46 & 75 & 96 & 88 \\
\hline$S 4$ & 82 & 96 & 47 & 86 & 56 & 98 & 86 & 87 \\
\hline$S 5$ & 42 & 45 & 49 & 55 & 78 & 82 & 98 & 97 \\
\hline S6 & 90 & 85 & 55 & 97 & 90 & 80 & 97 & 58 \\
\hline S7 & 90 & 84 & 54 & 97 & 98 & 82 & 84 & 45 \\
\hline$S 8$ & 86 & 88 & 95 & 68 & 97 & 97 & 98 & 78 \\
\hline
\end{tabular}

Table 5. PCA with $30 \times 30$ subspace

KNN classifier trained with PCA Features with 30 X30 subspace dimensions

\begin{tabular}{|c|c|c|c|c|c|c|c|c|}
\hline \multirow[t]{2}{*}{ Training } & \multicolumn{8}{|c|}{ Testing } \\
\hline & S1 & $S 2$ & $S 3$ & $S 4$ & $S 5$ & S6 & $S 7$ & S8 \\
\hline$S 1$ & 74 & 5 & 18 & 4 & 6 & 5 & 4 & 3 \\
\hline$S 2$ & 10 & 70 & 8 & 7 & 6 & 5 & 10 & 7 \\
\hline S3 & 6 & 8 & 72 & 9 & 10 & 6 & 8 & 11 \\
\hline$S 4$ & 8 & 9 & 10 & 76 & 10 & 9 & 8 & 7 \\
\hline$S 5$ & 6 & 5 & 4 & 4 & 72 & 10 & 11 & 12 \\
\hline S6 & 10 & 9 & 8 & 6 & 12 & 73 & 10 & 11 \\
\hline S7 & 12 & 14 & 15 & 16 & 15 & 16 & 70 & 18 \\
\hline$S 8$ & 18 & 17 & 10 & 11 & 13 & 12 & 11 & 72 \\
\hline
\end{tabular}

Table 6. TPCA with $30 \times 30$ subspace

KNN classifier trained with TPCA Features with $30 \times 30$ subspace dimensions

\begin{tabular}{|c|c|c|c|c|c|c|c|c|}
\hline \multirow[t]{2}{*}{ Training } & \multicolumn{8}{|c|}{ Testing } \\
\hline & $S 1$ & $S 2$ & S3 & $S 4$ & 55 & S6 & 57 & S8 \\
\hline$S 1$ & 78 & 45 & 53 & 57 & 62 & 60 & 61 & 59 \\
\hline$S 2$ & 66 & 65 & 64 & 66 & 62 & 61 & 69 & 68 \\
\hline$S 3$ & 40 & 65 & 64 & 62 & 61 & 53 & 54 & 53 \\
\hline$S 4$ & 42 & 58 & 62 & 67 & 68 & 63 & 62 & 60 \\
\hline$S 5$ & 39 & 44 & 60 & 61 & 65 & 68 & 62 & 60 \\
\hline S6 & 46 & 70 & 68 & 68 & 62 & 66 & 62 & 53 \\
\hline$S 7$ & 42 & 70 & 63 & 66 & 65 & 62 & 61 & 68 \\
\hline$S 8$ & 40 & 68 & 63 & 62 & 66 & 62 & 61 & 64 \\
\hline
\end{tabular}

Table 7. PCA with $20 \times 20$ subspace (Brute force method)

KNN classifier trained with PCA Features with $20 \times 20$ subspace dimensions (Brute Force Method)

\begin{tabular}{|c|c|c|c|c|c|c|c|c|}
\hline \multirow{3}{*}{ Training } & \multirow{2}{*}{\multicolumn{8}{|c|}{ Testing }} \\
\hline & & & & & & & & \\
\hline & $S 1$ & $S 2$ & S3 & $S 4$ & $S 5$ & 56 & $S 7$ & $S 8$ \\
\hline$S 1-S 2$ & 70 & 72 & 10 & 13 & 14 & 9 & 4 & 8 \\
\hline$S 2-S 3$ & 10 & 74 & 72 & 14 & 6 & 9 & 8 & 10 \\
\hline S3-S4 & 10 & 7 & 71 & 75 & 9 & 10 & 8 & 10 \\
\hline$S 4-S 5$ & 5 & 10 & 4 & 73 & 72 & 9 & 8 & 10 \\
\hline$S 5-S 6$ & 11 & 10 & 12 & 8 & 70 & 72 & 4 & 6 \\
\hline $56-57$ & 7 & 8 & 10 & 5 & 6 & 72 & 71 & 8 \\
\hline$S 7-S 8$ & 4 & 7 & 9 & 8 & 6 & 5 & 71 & 70 \\
\hline$S 8-S 1$ & 70 & 2 & 10 & 6 & 5 & 8 & 9 & 70 \\
\hline
\end{tabular}




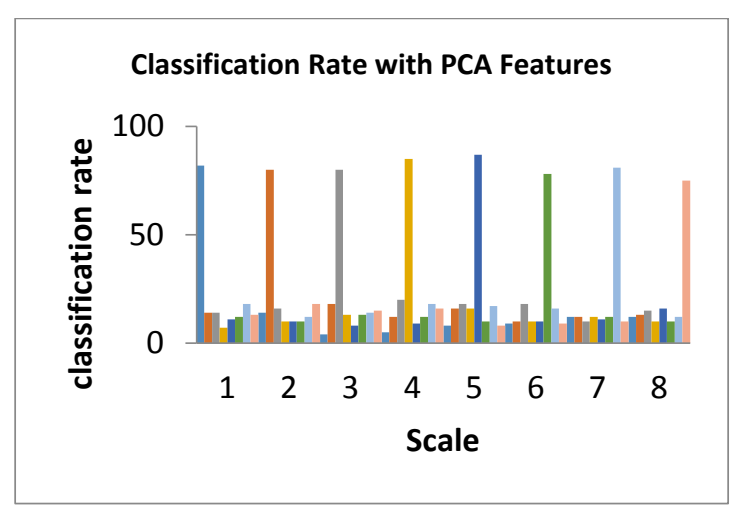

Fig.2. Plot of Classification rates with PCA features for 20x20 subspace

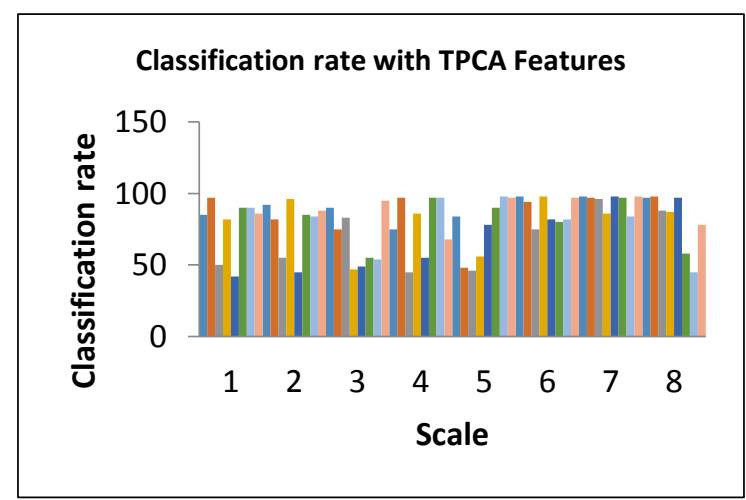

Fig.3. Plot of Classification rates with TPCA features for 20x20 subspace

\section{CONCLUSION AND DiscUSSION}

In this paper, we have model face recognition at a distance as a transfer subspace learning problem. By doing so we have shown that there is significant increase in the classification rate of cross scale samples. Brute force approach gives slightly lower results than TPCA approach. Brute force approach demands that all the samples in database should be made available at the time of training. However, in TPCA approach only a reference image of new samples id to be made available for training. . In the future, certain research issues need to address. Negative transfer can happen if the source and target domains are not related to each other in some sense. Negative transfer may cause the system to perform worse. To avoid negative transfer, we first need to find a measure of transferability.

We have experimented using PCA with different subspace dimensions viz. 10x10, 20x20, 30x30, 40x40 and 50x50 .Results upto 30x30 dimensions are listed in this paper. We found that as the subspace dimensions increase, the correlation gets captured which results in the decrease of classification rate. The best results are available with $20 \times 20$ subspace dimensions as maximum variance is captured by PCA in that subspace dimension. The results of the same are shown in Fig 2 and Fig 3.

\section{REFERENCES}

[1] Sinno Jialin Pan and Qiang Yang A Survey on Transfer Learning. IEEE Transactions on Knowledge and Data Engineering, 2010, vol 22, No 10.

[2] Si Si and Dacheng Tao Bregman Divergence -Based Regularization for Transfer Subspace Learning . IEEE Transactions on Knowledge and Data Engineering, 2010, vol22, No 7.

[3] M.Beklin, P.Niyogi and V.Sindhwani, Manifold Regularization: A Geometric Framework for Learning from Labeled and Unlabeled Examples. J.Machine Learning Research, 2006, 7:.2399-2434.

[4] B.Zadrozny Learning and Evaluating Classifiers under Sample Selection Bias. Proc. $21^{\text {st }}$ International conference on Machine Learning, 2004, 114-121.

[5] S.J.Pan, J.T. Kwok, and Q.Yang. Transfer Learning via Dimensionality reduction.Proceedings of $23^{\text {rd }}$ national conference on Artificial intelligence, 2008, 677-682

[6] P.N.Belhumeurb, J.P Hespanha, and D.J.Kriegman. Eigenfaces versus Fisherfaces: Recognition Using Class Specific Linear Projection," IEEE Transaction Pattern Analysis and Machine Intelligence, 1997, 19:711-720

[7] P. J. Phillips, H. Wechsler, J. Huang, and P. Rauss. The FERET database and evaluation procedure for face recognition algorithms. Image Vis. Comput. J., 1998, vol. 16.5: 295-306.

[8] Stan Z.Li and Anil K,.Jain . Handbook of Face Recognition, second Edition.Springer.

[9] Desislava Boyadzieva George Gluhchev. Neural Network and $\mathrm{KNN}$ classifier for on line signature verification. Lecture Mnotes in Computer Science, 2014, 8897:198206

[10] Ming Shao Dmitry Kit Yun Fu. Generalized Transfer subspace learning through low rank constraint. Int $\mathbf{J}$ Comput Vis. 2014, 109:74-93.

[11] Zheng Ming Ding, Ming Shao and Yun Fun, "Missing Modality Transfer Learning via latent low rank constraint" IEEE transactions on Image Processing, Nov 2015, vol 24, pp 4322-4334.

[12] Li Yang, Liping Jing and Michael K Ng, “ Robust and Non-negative Collective Matrix Factorization for Text-toImage Transfer Learning”, IEEE transactions on Image Processing, Dec 2015, vol 24, No 12, pp 4701-4714.

[13] Zhaohong Deng, Yizhang Jiang, "Generalized HiddenMapping Ridge Regression, Knowledge-Leveraged Inductive Transfer Learning for Neural Networks, Fuzzy Systems and Kernel Methods", IEEE Transactions on Cybernetics, Dec 2014, vol 44, No 12, pp 2585-2599

[14] Teddy Salan, Khan M Iftekharuddin, "Large Pose Invariant Face Recognition using feature based recurrent neural network", International Conference on Neural Network, 2012, DOI:10:1109/IJCNN.2010.6252795

[15] H Ebrahim Pour- Komleh, V Chandran, S Sridharan, "Robustness to expression variations in fractal base face recognition", Sixth International Symposium on Signal Processing and its Applications, 2001, vol 1, pp 359-362

[16] Horst Eidenberger, "Illumination Invariant Face Recognition by Kalman Filtering", Proceedings ELMAR 2006, DOI:10:1109/ELMAR.2006.329517

[17] M.S.Shashi Kumar, K.S.Vimala, N.Avinash, "Face Recognition distance estimation from a monocular camera", IEEE conference on Image Processing, 2013, DOI 10:1109/ICIP.2013.6738729 
[18] Fisher RA, "The use of multiple measurements in taxonomic problems, Ann Evgen, 7(2), 1936, pp 179-188

[19] He X, Niyogi P, "Locality preserving projections", Advanced Neural Information Processing System, 2003, pp 16:1-8

[20] Belhumeur p, Hespanha J, Kriegman D, "Eigenfaces vs fisher faces: recognition using class specific linear projection, IEEE Trans Pattern Analysis and Machine Intelligence, 1997, 19(7), pp 711-720

\section{Authors' Profiles}

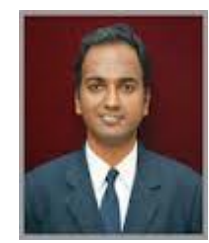

Alwin Anuse received the B.E and M.E degrees from Pune University. He is currently pursuing Ph.D. degree from College of Engineering Pune. His research areas are Neural Networks, signal Processing etc.

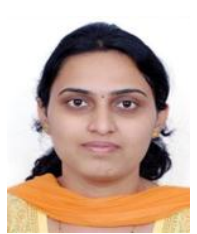

N. A. Deshmukh received the B.E. degree in Electronics and M.E. degree in ElectronicsDigital Systems from University of Pune in 2002 \& 2013 respectively; she secured first rank in M.E. Electronics at MIT, Pune. She worked as NOC Engg. at Tulip Telecom Pvt.Ltd. in 2006. She was Visiting Lecturer at MIT,Pune in the Department of Computer Engg. and E \& TC
Engg. from 2009 to 2013. She is currently Assistant Professor in the Department of E \& TC Engg. at AISSMS IOIT, Pune.

Her research interests include Machine Learning, Computer Vision \& Pattern Recognition.

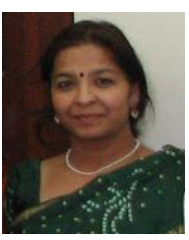

Vibha Vyas did her BE from Nagpur university in 1995, M.Tech in 2002 and $\mathrm{PhD}$ in 2010 from COEP. Her area of interest are Signal and Image processing with various applications of machine vision and multidimensional processes. She is guiding various UG and PG level students in said area.

How to cite this paper: Alwin Anuse, Nilima Deshmukh, Vibha Vyas,"Transfer Subspace Learning Model for Face Recognition at a Distance", International Journal of Image, Graphics and Signal Processing(IJIGSP), Vol.9, No.1, pp.27-32, 2017.DOI: 10.5815/ijigsp.2017.01.04 